\title{
Comparaison des ectomycorhizes naturelles entre le hêtre (Fagus sylvatica) et 2 lactaires (Lactarius blennius var viridis et Lactarius subdulcis). I. Caractéristiques morphologiques et cytologiques.
}

\author{
A Prévost, JC Pargney * \\ Université de Nancy I, faculté des sciences, laboratoire de biologie des ligneux, \\ BP 239, 54506 Vandouvre cedex, France
}

(Reçu le 5 avril 1994; accepté le 28 juin 1994)

\begin{abstract}
Résumé - Lactarius blennius var viridis et Lactarius subdulcis forment avec les racines du hêtre des ectomycorhizes lisses qui présentent des différences importantes au niveau de leur organisation : les manteaux montrent des structures différentes et le réseau de Hartig, normalement développé chez L subdulcis, est discontinu dans iautre espèce. Chez les 2 mycorhizes, des laticifères sont abondants dans le manteau et des hyphes intracellulaires se développent dans les cellules racinaires du hêtre. L'ectomycorhize à $L$ subdulcis est fréquemment infectée par un ascomycète dont les hyphes sont présentes tant au niveau du manteau que dans le cortex racinaire.
\end{abstract}

ectomycorhize / Lactarius / Fagus sylvatica / morphologie / ultrastructure

Summary - Comparison of natural ectomycorrhizas between beech (Fagus sylvatica) and 2 fungi (Lactarius blennius var viridis and Lactarius subdulcis). I. Morphological and cytological characteristics. Lactarius blennius var viridis and $L$ subdulcis form smooth ectomycorrhizas with beech roots. These mycorrhizas present large differences in organization. Fungal mantles show different structures. The Hartig net is normally developed in L subdulcis and is discontinuous in the other species. Both mycorrhizas contain many laticifers and intracellular hyphae in the root cell of beech. $L$ subdulcis mycorrhizas are frequently infected by an ascomycete whose hyphae are present equally in the manthe and the root cortex.

ectomycorrhiza / Lactarius / Fagus sylvatica / morphology / ultrastructure

* Correspondance et tirés à part 


\section{INTRODUCTION}

Selon les plantes hôtes, la mycorhization peut être plus ou moins importante. Le hêtre présente un très fort taux de mycorhization puisque $100 \%$ des racines courtes correspondent à des mycorhizes. Celles-ci sont de couleur, de taille et de morphologie très variées. Elles peuvent être lisses et de couleur orange, brune ou noire, montrer un manteau hérissé de soies blanches ou noires, caractéristiques de Cenococcum, présenter un mycélium frangeant formant un feutrage blanc crème ou un mycélium cotonneux doré, porter des cordons ou des spinules comme chez les Tuber (Prévost, 1992).

Les mycorhizes lisses sont élaborées essentiellement par des lactaires et des russules. Les laticifères fonctionnels qui caractérisent les carpophores des lactaires sont également présents dans les manteaux des mycorhizes édifiées à partir de ces champignons. Diverses études cytologiques ont permis de mieux connaitre la structure de ces mycorhizes (Luppi et Gautero, 1967 ; Voiry, 1981 ; Münzenberger et al, 1986 ; Agerer, 1987 ; Brand, 1993).

Dans le présent travail, nous nous proposons de comparer 2 mycorhizes de lactaires fréquentes chez le hêtre en décrivant les aspects macroscopiques et cytologiques qui permettent de dégager les points communs aux 2 mycorhizes et des particularités propres à l'une et à l'autre. Cette première approche est le début d'un travail conduisant à une meilleure connaissance du fonctionnement des mycorhizes de lactaires. Elle sera ultérieurement suivie et complétée par des études plus spécifiques concernant la cytophysiologie des interfaces hêtre-lactaires, les relations mycorhize-sol par l'intermédiaire des rhizomorphes et par l'étude du suivi de l'évolution saisonnière des 2 mycorhizes.

\section{MATÉRIEL ET MÉTHODES}

Des racines de hêtre sont prélevées sur des arbres adultes âgés de 80 à 100 ans dans une hêtraie située en forêt de Haye près de Nancy (Meurthe-et-Moselle) et dans les bois de Vauvillers (Haute-Saône) à une centaine de kilomètres de Nancy dans une chênaie-hêtraie. Les 2 stations sont sur sol brun lessivé. Les prélèvements sont réalisés aux pieds des arbres, là où se trouvent une multitude de racines courtes dont la présence pourrait être due à un écoulement le long du tronc des précipitations interceptées (Aussenac, 1975, cité par Riedacker, 1981).

\section{Techniques de microscopie électronique à balayage}

Des racines portant des mycorhizes sont fixées par le glutaraldéhyde à $2,5 \%$ dans le tampon cacodylate à $\mathrm{pH} 7,2$ pendant $2 \mathrm{~h}$, rincées dans le tampon, puis post-fixées par tétroxyde d'osmium $0,5 \%$ dans le tampon pendant $1 \mathrm{~h}$.

Après rinçage par l'eau distillée, déshydratation par l'acétone et passage au point critique, les échantillons subissent une métallisation à l'or et sont observés sur un microscope électronique à balayage "Hitachi $S 2500$ ".

\section{Techniques de cytologie photonique}

Des coupes transversales réalisées à main levée sont colorées pendant $15 \mathrm{~min}$ par le réactif de Lugol afin de visualiser le latex fongique (Luppi et Gautero, 1967 ; Voiry, 1981).

Des coupes semi-minces $(200$ à $300 \mathrm{~nm}$ d'épaisseur) sont réalisées à partir des objets destinés à l'observation en microscopie électronique à transmission. Recueillies sur lame de verre, elles sont colorées par le bleu de toluidine à $1 \%$ dans $2,5 \%$ de $\mathrm{Na}_{2} \mathrm{CO}_{3}(\mathrm{pH} 11,6)$. Elles permettent de contrôler l'état de mycorhization des racines avant l'observation en cytologie ultrastructurale.

Les coupes semi-minces peuvent être également colorées par le Soudan III, colorant des lipides et des substances résinifères (Dop et Gautier, 1928 ; Lison, 1960) et qui marque également le latex des plantes supérieures (de Faÿ, 
1981). Après traitement par le Soudan III et lavage, les coupes sont colorées par le bleu de toluidine afin de visualiser les tissus fongiques et racinaires.

\section{Microscopie électronique à transmission}

Les mycorhizes sont coupées longitudinalement sous la loupe binoculaire dans une goutte de fixateur (glutaraldéhyde à $2,5 \%$ dans un tampon phosphate $0,1 \mathrm{M}$ à pH 7,2 ou cacodylate $0,1 \mathrm{M}$ au même $\mathrm{pH}$ ). Selon leur taille, elles sont débitées en 2 ou 4 morceaux. Certaines mycorhizes ont ainsi pu être étudiées jusqu'à $20 \mathrm{~mm}$ de l'apex.

Les objets sont fixés pendant $6 \mathrm{~h}$ à la température de la glace fondante. Après plusieurs rinçages par le tampon de fixation, ils sont mis à rincer au froid toute une nuit dans le même milieu.

La post-fixation est effectuée pendant $1 \mathrm{~h}$ par le tétroxyde d'osmium à $2 \%$ dans le tampon utilisé initialement.

Les échantillons sont soigneusement rincés à l'eau distillée, déshydratés par des bains d'acétone en concentrations croissantes, puis imprégnés dans la résine de Spurr. Afin d'obtenir une meilleure inclusion, l'imprégnation est de longue durée (minimum $20 \mathrm{j}$ ).

Des coupes minces (60 à $80 \mathrm{~nm}$ ), réalisées sur un ultramicrotome Porter Blum, sont recueillies sur des grilles en cuivre-rhodium. Les coupes sont contrastées par l'acétate d'uranyle et le citrate de plomb. Elles sont observées au microscope électronique Zeiss 902.

\section{RÉSULTATS}

\section{Étude morphologique}

\section{L'ectomycorhize à Lactarius blennius var viridis (planche 1, fig 1)}

Elle est principalement récoltée dans l'humus et dans l'horizon $\mathrm{A} 1$ mais très rarement plus profondément.
C'est une mycorhize orangée, ramifiée, pyramidale, lisse, à apex clair, de type C1 selon la classification de Voiry (1981). Quelques hyphes émanent parfois du manteau. De même couleur que la mycorhize, des rhizomorphes s'y rattachent. Les laticifères forment un réseau blanc visible par transparence.

En microscopie électronique à balayage, la mycorhize montre une surface bosselée (planche 1, fig 10). Cet aspect du manteau paraît constant sur toute la mycorhize. Les craquellements préserits localement correspondent à de nouvelles ramifications en cours de formation (planche 2, fig 11). Des hyphes sont présẻntes à la surface du manteau (planche 2, fig 11).

\section{L'ectomycorhize à Lactarius subdulcis (planche 1, fig 6)}

Elle est principalement récoltée dans l'horizon A1, quelquefois dans l'horizon A2, mais rarement dans l'humus. Elles ne cohabitent jamais avec d'autres mycorhizes.

C'est une mycorhize jaune orangé, très ramifiée, lisse, à apex légèrement plus clair, de type $\mathrm{C} 1$ selon la classification de Voiry (1981). Quelques hyphes émanent du manteau. Des rhizomorphes de même couleur que la mycorhize y sont parfois associés. Les laticifères sont difficilement visibles par transparence. Les très jeunes mycorhizes sont beaucoup plus claires que les mycorhizes âgées dont elles sont issues. Les mycorhizes de grande taille prennent une couleur brune à leur base.

En microscopie à balayage, la surface du manteau montre une juxtaposition d'hyphes de grande taille, plus ou moins écrasées (planche 2, fig 12). Cette structure est présente sur toute la mycorhize. Des hyphes isolées ou des groupes d'hyphes émanent du manteau (planche 2, fig 13). 

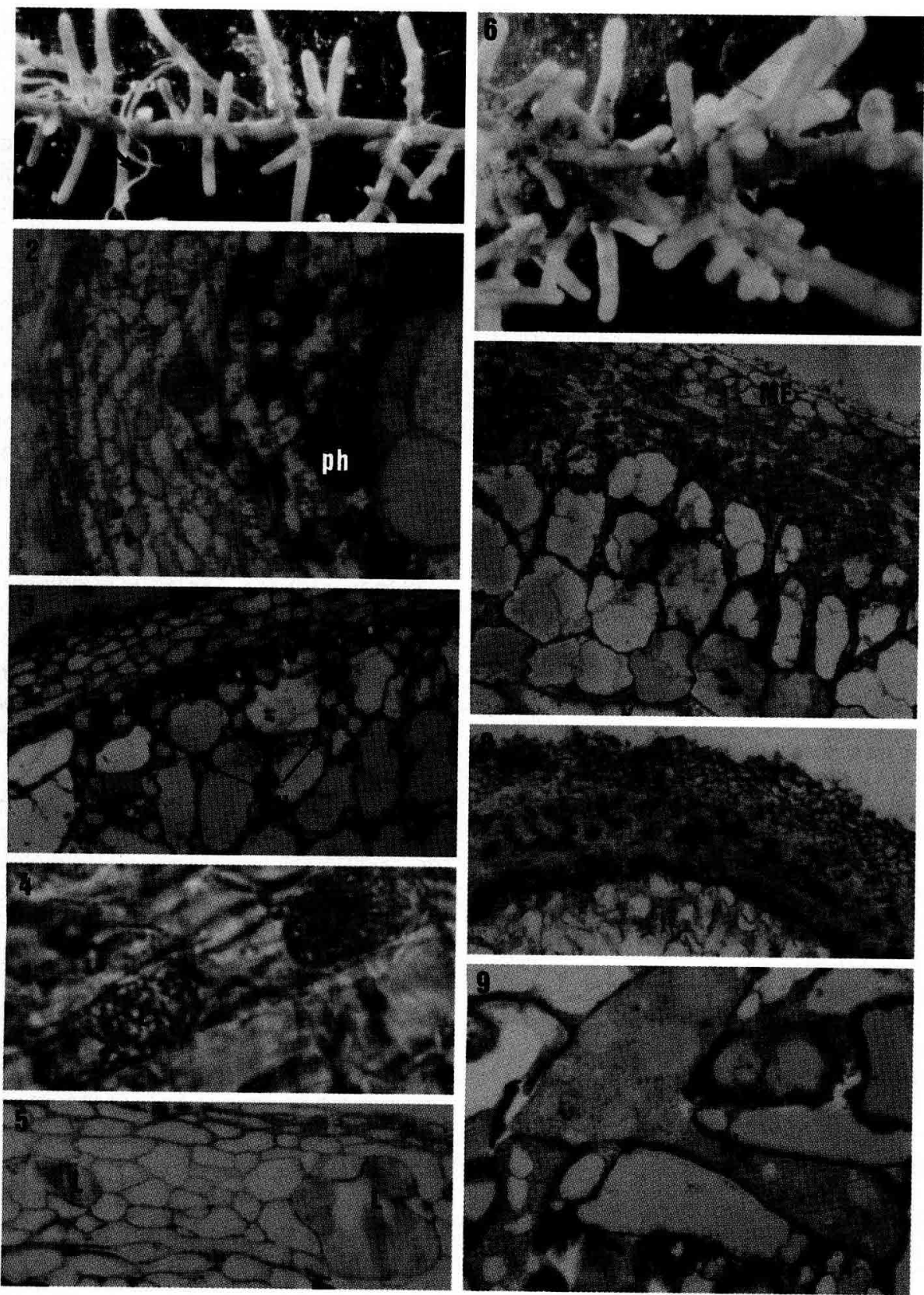


\section{Étude en microscopie photonique}

\section{L'ectomycorhize à Lactarius blennius var viridis}

Elle montre un manteau limité extérieurement par une substance qui se colore par le bleu de toluidine (planche 1, figs 2 et 3 ). Les strates les plus internes du manteau présentent des plages sombres correspondant à des composés polyphénoliques particulièrement importants à l'apex de la mycorhize (planche 1, fig 2). Les laticifères sont mis en évidence dans le manteau, par le réactif de Lugol (planche 1, fig 4) et par le rouge Soudan III (planche 1, fig 5).

Le réseau de Hartig apparaît discontinu (planche 1, fig 3) ; il est présent jusqu'à la troisième couche de cellules corticales.

\section{L'ectomycorhize à Lactarius subdulcis}

Elle montre un manteau composé de 2 parties (planche 1, fig 7). À l'extérieur, les hyphes sont grosses et à contenu clair ; à l'intérieur, elles sont de petite taille et ont un contenu dense. Le manteau est séparé des cellules corticales par des couches de composés polyphénoliques plus nombreuses à l'apex que dans les autres parties. L'utilisation du réactif de Lugol met en évidence la présence de laticifères dans le manteau interne des mycorhizes (planche 1, fig 8).
Le réseau de Hartig est bien visible et peut s'insinuer jusqu'à la troisième couche de cellules corticales (planche 1, fig 7). II est continu et unisérié.

Des hyphes d'un champignon ascomycète, reconnaissable à la présence de corps de Woronin de part et d'autre des septa (planche 1, fig 9), sont associées aux mycorhizes.

\section{Étude en microscopie électronique à transmission}

\section{L'ectomycorhize à Lactarius blennius var viridis}

Le manteau est de type pseudoparenchymateux. Sur les coupes longitudinales comme sur les coupes transversales, les hyphes montrent des diamètres très différents (planche 3 , fig 4). Le manteau se différencie en plusieurs zones.

Extérieurement il est limité par une substance, peu opaque aux électrons, qui isole les hyphes de la rhizosphère (planche 3 , fig 14 ; planche 4, fig 16). Des hyphes de petite section y sont incluses. Le plus souvent dégénérescentes, elles sont parfois vivantes et plurinuclées (planche 4, fig 16).

Sous cette zone externe, les hyphes montrent progressivement des sections qui s'agrandissent, d'une part, et, d'autre part, des interfaces qui s'amincissent (planche

Planche 1. Fig 1. Macrophotographie de la mycorhize à $L$ blennius portant des rhizomorphes (flèches). Figs 2-5. Coupes semi-minces de la mycorhize à $L$ blennius. 2. Coupe dans la zone apicale : le manteau limité extérieurement par une substance colorée par le bleu de toluidine $(S)$ et intérieurement par des polyphénols $(\mathrm{ph})$ renferme des laticifères $(L)(\times 1600)$. 3. Coupe dans une zone sous-apicale : le réseau de Hartig apparaît discontinu $(\mathrm{RH})(\times 650)$. 4. Latex des laticifères $(L)$ coloré par le réactif de Lugol (x 2500). 5. Latex des laticifères $(L)$ coloré par le Soudan III (x 1600). Fig 6. Macrophotographie de la mycorhize à $L$ subdulcis. Figs 7-9. Coupes semi-minces de la mychorhize à $L$ subdulcis. 7 . Coupe d'une zone sous-apicale montrant les 2 zones du manteau (ME et MI) et le réseau de Hartig (RH) continu $(\times 500)$. 8. Coupe dans la zone apicale, colorée par le réactif de Lugol : les laticifères $(L)$ sont nombreux dans la manteau interne $(x 250)$. 9. Des hyphes d'ascomycète reconnaissables par les grains de Woronin $(W)$ sont associées à la mycorhize $(x 1600)$. 

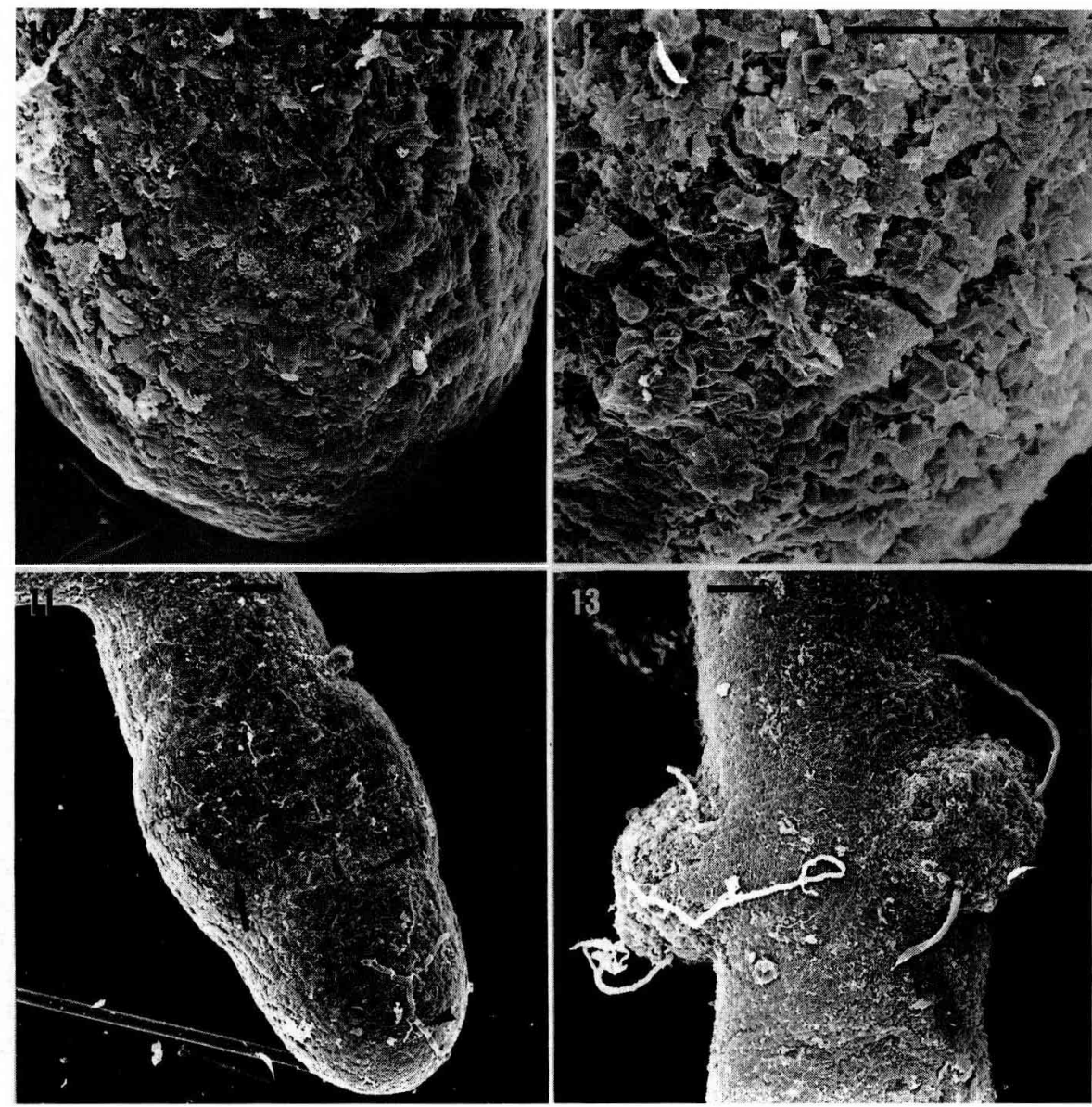

Planche 2. Figs 10-11. Mycorhize à $L$ blennius. 10. Aspect de la surface du manteau apical (x 500). 11. Craquellements du manteau dans la zone latérale de la mycorhize (flèches) et hyphes de la surface du manteau (tête de flèche) (x 180). Figs 12-13. Mycorhize à $L$ subdulcis. 12. Aspect de la surface du manteau apical $(x 700)$. 13. Jeunes ramifications et hyphes émanants du manteau $(x 180)$. Les traits correspondent à $50 \mu \mathrm{m}$.

3 , fig 14). Leur cytoplasme très vacuolisé peut renfermer de nombreuses vésicules le long des parois (planche 4, fig 17). Cette zone intermédiaire est limitée intérieurement par des hyphes de taille réduite (planche 4, fig 14) très vacuolisées (planche 4 , fig 18).

Le manteau interne est constitué d'hyphes vivantes peu vacuolisées et de taille réduite (planche 4 , fig 14). Les inter- 


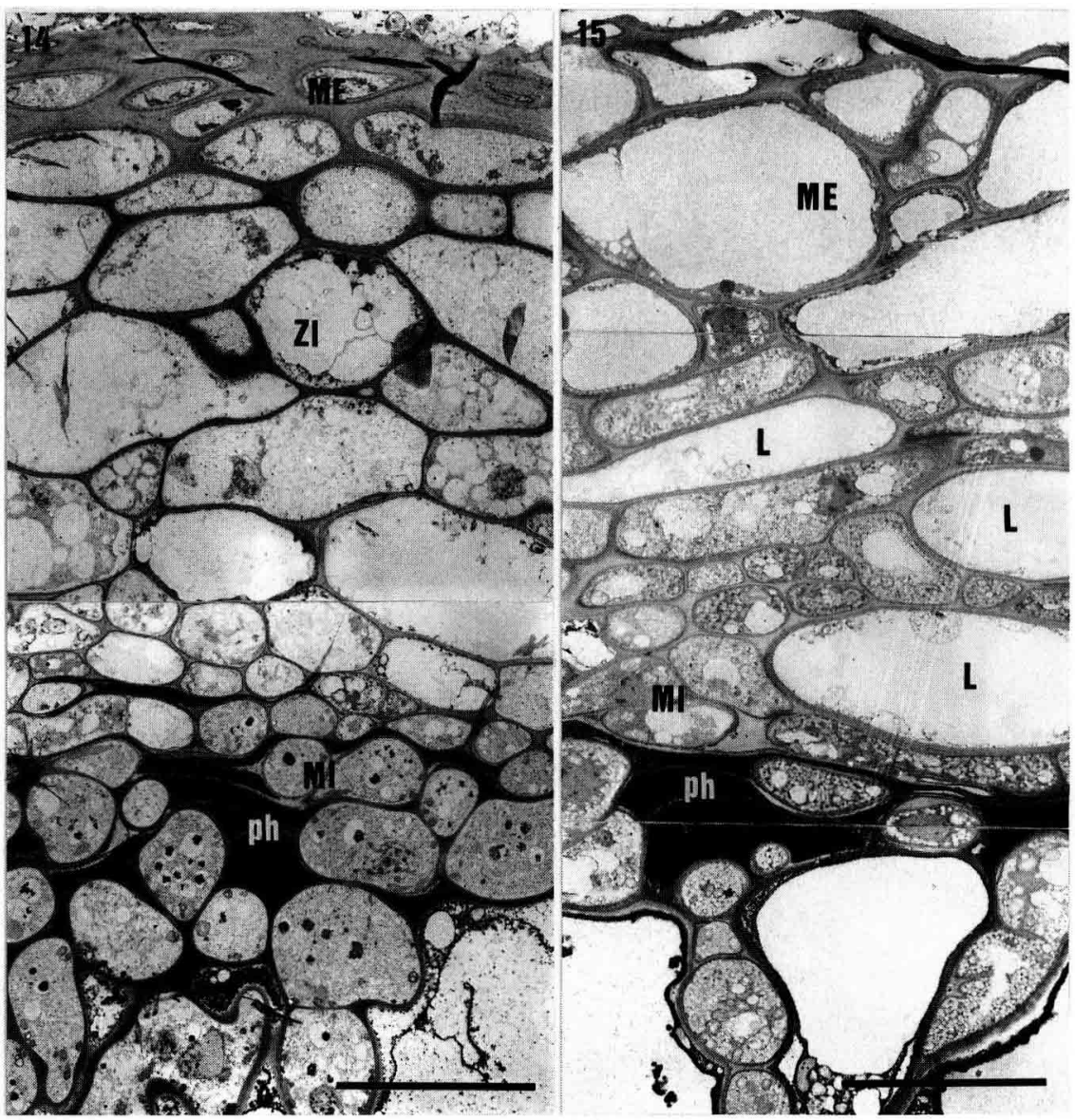

Planche 3. Fig 14. Coupe transversale dans la manteau à $L$ blennius. La transition entre le manteau externe (ME) et le manteau interne (MI) s'effectue progressivement par une zone intermédiaire (ZI). Des bandes de composés polyphénoliques (ph) sont présentes dans le manteau interne (x 3000$)$. Fig 15. Coupe transversale dans le manteau à $L$ subdulcis. La séparation entre le manteau externe (ME) et le manteau interne (MI) est nette. Des laticifères, ici dépourvus de contenu cellulaire ( $L$ ), et des composés polyphénoliques (ph) sont présents dans le manteau interne (x 3000). Les traits correspondent à $10 \mu \mathrm{m}$.

faces sont plus fines que celles des hyphes des autres zones. D'étroites bandes de composés polyphénoliques sont présentes dans le manteau interne. Elles s'insèrent entre les hyphes ou forment des empilements dans lesquels chaque place reste séparée des autres par de fines parois moins denses aux électrons. Les formes multiples des 


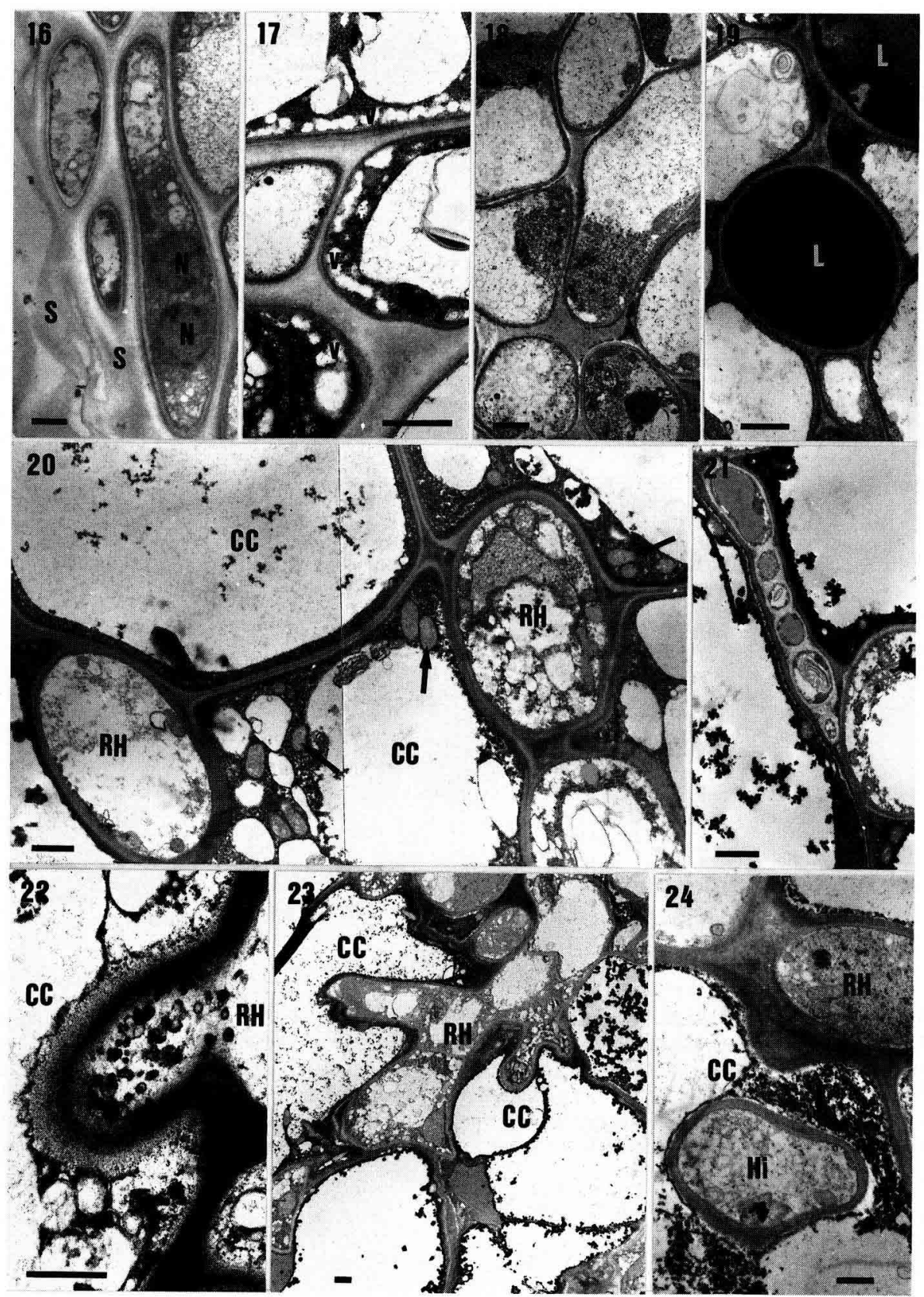


bandes polyphénoliques sont le reflet des contraintes exercées par les hyphes (planche 4, fig 14).

Les laticifères sont présents à différents niveaux du manteau (zones intermédiaire et interne). Les grandes vacuoles renferment du latex opaque aux électrons et repoussent en position pariétale le cytoplasme dont la densité électronique rend difficile l'observation d'organites (planche 4, fig 19).

Le réseau de Hartig est discontinu (planche 4, fig 20). Cependant les hyphes sont parfois séparées par une succession d'articles de très petite section et qui pourraient correspondre à des ramifications d'une même hyphe (planche 4, fig 21). Le réseau peut également émettre des digitations en direction des cellules corticales dégénérescentes (planche 4, fig 22) qui peuvent aboutir à des pénétrations dans les cavités cellulaires (planche 4, figs 23 et 24).

Les cellules corticales sont très vacuolisées. Leur cytoplasme peut renfermer quelques organites : mitochondries, réticulum endoplasmique (planche 4, fig 20). II est souvent réduit à une étroite bande pariétale et dense, dans laquelle les structures sont difficilement identifiables (planche 4, figs 20 et 21 ). Des composés polyphénoliques peuvent s'accumuler dans les vacuoles, sous forme de granulations denses localement condensées (planche 4, fig 23).

\section{L'ectomycorhize à Lactarius subdulcis}

Le manteau se différencie en 2 zones : l'une interne, l'autre externe. Le manteau externe est pseudoparenchymateux et constitué de 4 ou 5 assises d'hyphes montrant parfois de grande section (planche 3 , fig 15). À l'apex ces hyphes sont vivantes (planche 5, fig 25), mais se vacuolisent rapidement et leur cytoplasme dégénère dans la région sous-apicale de la mycorhize (planche 3, fig 15). Le manteau interne est prosenchymateux et constitué de 5 à 10 assises d'hyphes présentant des sections variées. Elles renferment de petites vacuoles et un cytoplasme important (planche 3, fig 15 ; planche 5, fig 27). Des vésicules bordent fréquemment le plasmalemme avec lequel elles paraissent être en contact (planche 5, fig 27).

Des composés polyphénoliques sont présents dans le manteau interne (planche 3 , fig 15). Ils forment des plages plus ou moins importantes qui sont fragmentées par la pénétration d'hyphes dont le contenu peut apparaître très dense (planche 5, fig 28).

Les laticifères montrent des sections allongées. Beaucoup sont dépourvus de contenu cellulaire (planche 3 , fig 15), mais, dans certains cas favorables, le latex est préservé et apparaît sous forme de globules clairs bordés de cytoplasme très dense aux électrons (planche 5, fig 29).

Planche 4. Figs 16-24. Mycorhize à $L$ blennius. 16. Les hyphes du manteau externe peuvent être vivantes et renfermer plusieurs noyaux $(N)$; elles sont incluses dans une substance (S) qui limite extérieurement le manteau (x 7000 ). 17. Grandes hyphes de la zone intermédiaire renfermant de nombreuses vésicules (v) le long des parois ( $x$ 13 000). 18. Petites hyphes vacuolisées de la zone intermédiaire ( $x 7000)$. 19. Laticifères $(L)$ de la zone intermédiaire à contenu dense aux électrons $(x 9000)$. 20. Aspect du réseau de Hartig discontinu $(\mathrm{RH})$; les cellules corticales $(\mathrm{CC})$ peuvent encore renfermer des organites identifiables (flèches) $(x \quad 8000)$. 21. Les grosses sections d'hyphes du réseau peuvent être séparées par des hyphes de petites sections (x 8000 ). 22. Amorce d'une pénétration d'hyphe du réseau $(\mathrm{RH})$ dans une cellule corticale $(\mathrm{CC})(\times 15000)$. 23. Hyphe du réseau $(\mathrm{RH})$ pénétrant dans les cellules corticales voisines $(\mathrm{CC})(\times 3000)$. 24. Hyphe intracellulaire $(H I)$ issue du réseau de $\mathrm{Hartig}(\mathrm{RH})$ et située dans une cellule corticale $(C C)$ de la première assise des cellules racinaires $(x 7000)$. Les traits correspondent à $1 \mu \mathrm{m}$. 

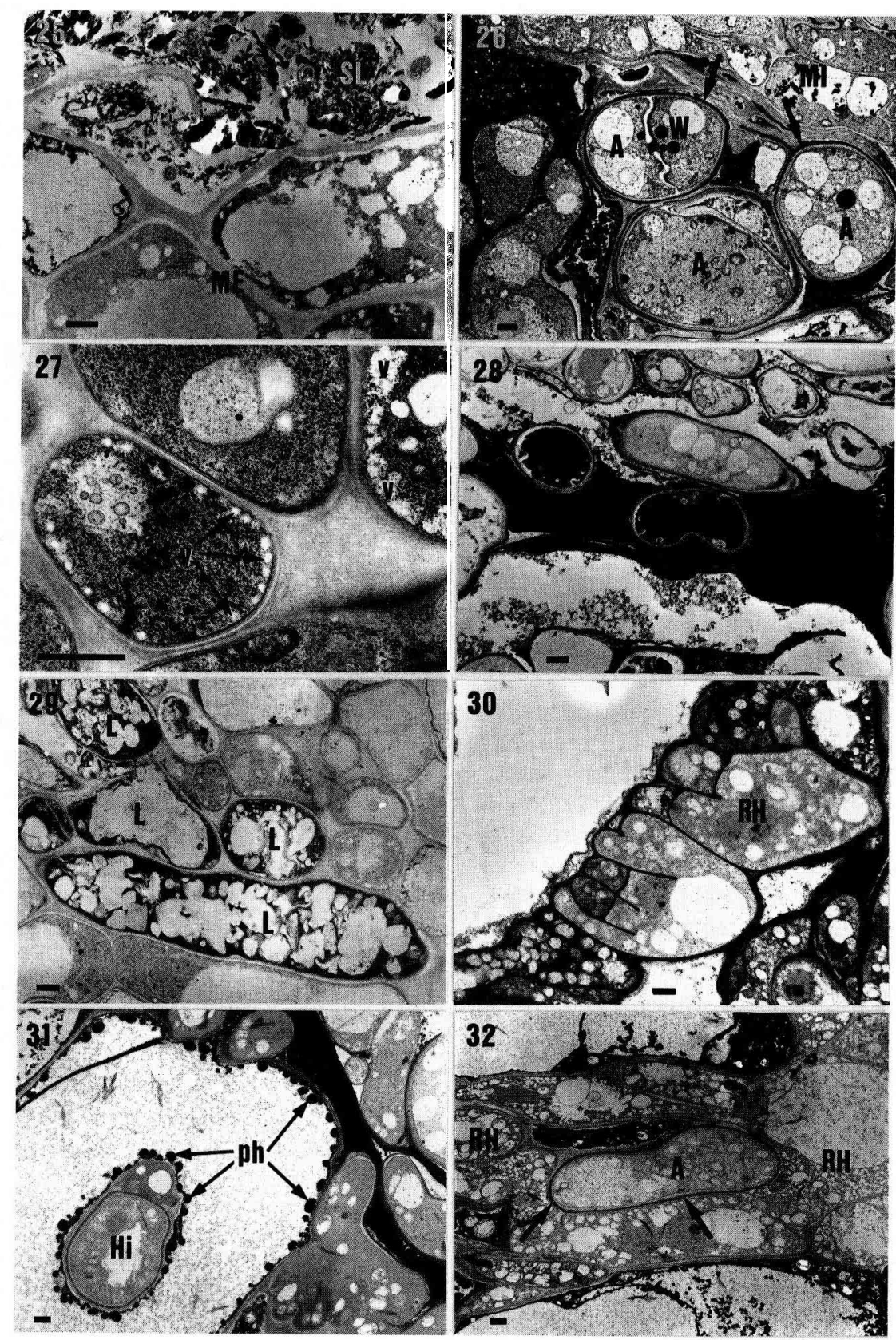
Des hyphes d'Ascomycète sont fréquemment observées dans le manteau interne, soit isolément, soit groupées (planche 5, fig 26). Elles sont reconnaissables par la présence de grains de Woronin et par l'existence d'une couche pariétale périphérique, dense aux électrons (planche 5, fig 26).

Le réseau de Hartig est continu, unisérié, formé d'hyphes vivantes (planche 3 , fig 15). Les coupes tangentielles au réseau montrent la présence d'invaginations pariétales et révèlent la complexité des structures fongiques intercellulaires (planche 5 , fig 30 ). Le réseau est bordé par des cellules corticales très souvent dégénérées dont le cytoplasme est réduit à une étroite bande pariétale, dense aux électrons (planche 3 , fig 15) et dont la vacuole peut renfermer des composés polyphénoliques soit épais, soit condensés en gouttelettes le long du tonoplaste (planche 5, fig 31).

Des hyphes d'Ascomycète sont également identifiables grâce aux grains de Woronin qu'elles renferment (planche 5, fig 32 ; planche 6 , fig 36 ), et à la couche pariétale périphérique (planche 5 , fig 32 ; planche 6 , fig 37 ). Elles possèdent de nombreux noyaux (planche 6, figs 34 et 35 ), d'abondantes vacuoles et de multiples organites (planche 6, figs 35 et 36). Elles occupent des positions variées : à l'apex entre des cellules corticales dépourvues de réseau (planche 6, fig 36) et dans les zones éloignées de l'apex au niveau du réseau (planche 5, fig 32) ou sous le réseau (planche 5, fig 33). Elles peuvent devenir intracellulaires par rupture des parois cellulaires (planche 6, fig 34) et envahir les cellules corticales tant à l'apex (planche 6, fig 37) que dans les zones plus éloignées (planche 6, figs 33 et 35). Dans les cellules apicales, la présence d'organites indique que le cytoplasme est vivant (planche 6, fig 37). Au niveau des zones éloignées de l'apex, les hyphes d'Ascomycète sont entourées par des dépôts denses issus de la dégénérescence du cytoplasme et de l'accumulation des polyphénols vacuolaires (planche 6, fig 35).

\section{DISCUSSION}

Les 2 mycorhizes sont lisses, sans ornementations. Le manteau de l'ectomycorhize à $L$ subdulcis est nettement composé de 2 parties, un manteau externe et un manteau interne alors que celui de l'ectomycorhize à $L$ blennius présente une structure différente avec un passage progressif du manteau externe à un manteau interne par une zone de transition.

Les hyphes du manteau externe de l'ectomycorhize à $L$ subdulcis, directement en contact avec le sol, sont souvent mortes et écrasées. Une structure similaire est également décrite chez les mycorhizes à $L$ deterrimus (Müzenberger et al, 1986 ; Agerer, 1987) et $L$ picinus (Agerer, 1987) asso-

Planche 5. Figs 25-32. Mycorhize à $L$ subdulcis. 25. Surface du manteau externe apical (ME) en contact avec les particules du sol (SL) (x 6000$)$. 26. Présence d'hyphes d'un Ascomycète (A) dans le manteau interne (MI) et reconnaissables par les grains de Woronin (W) et une couche pariétale dense (flèches) (x 4 000). 27. Hyphes du manteau interne montrant des vésicules (v) le long de la paroi (x 16000$)$. 28. Les composés polyphénoliques du manteau interne peuvent être traversés par des hyphes à contenu très dense (x 4 000). 29. Laticifères $(L)$ dans le manteau interne (x 4000$) .30$. Coupe tangentielle du réseau de Hartig (RH) (x 4000$)$. 31. Hyphe intracellulaire (HI) ; la cellule corticale renferme des polyphénols ( $\mathrm{ph}$ ) sous forme de gouttelettes (x 3000$)$. 32. Hyphe d'Ascomycète (A) séparée des hyphes du réseau de Hartig $(\mathrm{RH})$ par une couche pariétale dense (flèches) $(x 3000)$. Les traits correspondent à $1 \mu \mathrm{m}$. 


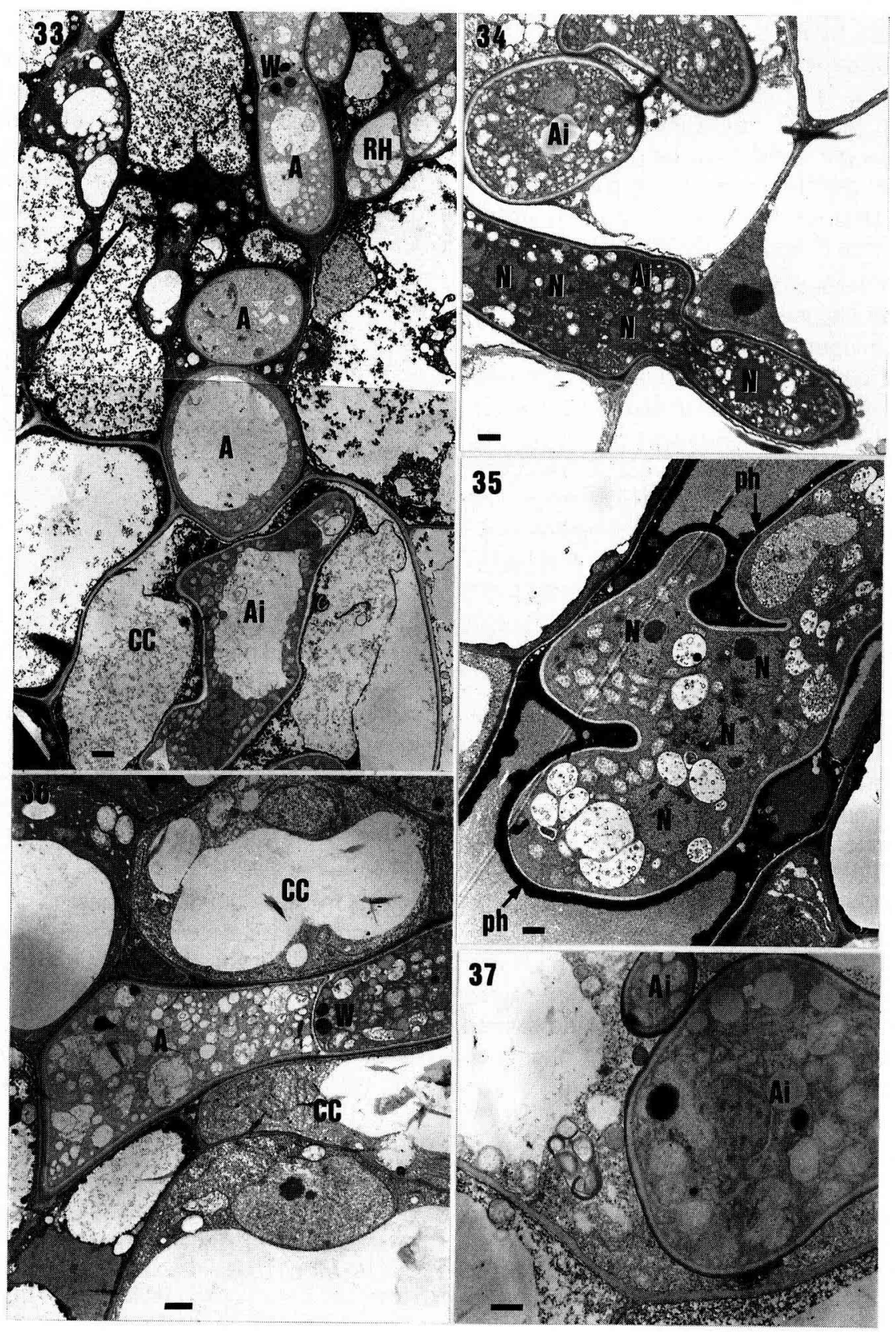


ciées à l'épicéa. En revanche, l'ectomycorhize à $L$ blennius est isolée du sol par du matériel qui pourrait être une production mucilagineuse des hyphes. La présence de mucilage est signalée dans de nombreuses mycorhizes à lactaires : chez $L$ flexuosus, $L$ piperatus, $L$ vellereus, $L$ chrysorrheus (Luppi et Gautero, 1967), chez $L$ volemus (Voiry, 1981), enfin chez $L$ vellereus (Brand, in Agerer, 1987). Une telle substance, réfringente et superficielle, est également décrite dans la mycorhize à Boletus edulis avec le chêne (Luppi et Gautero, 1967) ; mais dans ce cas la couche mucilagineuse est traversée par de nombreuses hyphes frangeantes.

Si Voiry (1981) pensait que la présence d'un mucilage est un caractère commun à toutes les mycorhizes de lactaires, nous montrons ici, comme Agerer (1987) et Brand (in Agerer, 1987), que le manteau des mycorhizes de lactaires peut présenter des structures différentes selon les espèces. II est vrai que lorsqu'une couche mucilagineuse est présente à la surface d'un manteau lisse, nous pouvons dire qu'il s'agit d'une mycorhize de lactaire, mais la réciproque n'est pas vérifiée.

Les laticifères sont inégalement répartis dans le manteau et ont un latex abondant et bien conservé par la fixation chez $L$ blennius; ils sont nombreux chez $L$ subdulcis, bien visibles en microscopie photonique, mais le latex s'observe difficilement en microscopie électronique à transmission du fait de sa mauvaise conservation lors de la fixation qui pourrait être due à une différence de fluidité par rapport au latex de $L$ blennius ; le latex se présente alors sous forme de masses alvéolées. Des images identiques ont été décrites par Müzenberger (1991) dans des laticifères de mycorhizes de $L$ deterrimus/Picea abies. Le latex des laticifères pourrait avoir un rôle protecteur. Le vélutinal, sesquiterpène instable, est un constituant majeur du latex des carpophores de plusieurs espèces de lactaires (FavreBonvin et al, 1982 ; Stemer et al, 1983, cités par Sterner et al, 1985), et les sesquiterpènes jouent un rôle protecteur contre les prédateurs (Sterne et al, 1985 ; Wicklow, 1988).

Dans les 2 mycorhizes étudiées, le réseau progresse entre 2, 3 ou 4 couches de cellules corticales ; il n'atteint jamais l'endoderme. Le réseau de Hartif formé par $L$ subdulcis est continu, unisérié, alors que celui formé par $L$ blennius est, au contraire, discontinu, très intimement lié aux structures pariétales des cellules corticales. Ils ne sont jamais présents au niveau apical. Des coupes tangentielles au réseau de Hartig montrent que les cloisons ou septums observés en coupes longitudinales sont en fait des replis de l'hyphe sur elle-même (Blasius et al, 1986 ; Kottke et Oberwinkler, 1986, 1987 ; Melville et al, 1988 ; Massicotte et al, 1990).

Des pénétrations intracellulaires sont souvent observées au niveau des premières cellules corticales mortes ou très dégénérescentes. Toutefois des organites peuvent

Planche 6. Figs 33-37. Mycorhize à $L$ subdulcis. 33. Les hyphes d'ascomycète peuvent être intercellulaires $(A)$ dans le réseau de Hartig $(\mathrm{RH})$ puis intracellulaires (Ai) dans une cellule corticale $(\mathrm{CC})$ sous le réseau; des grains de Woronin $(W)$ sont visibles (x 4000$)$. 34. Hyphes intracellulaires d'ascomycète (Ai) passant de cellule à cellule en subissant un étranglement flèches); de nombreux noyaux $(N)$ sont visibles $(x 4000)$. 35. Hyphe intracellulaire d'Ascomycète entourée par d'abondants dépôts polyphénoliques $(\mathrm{ph})$; là aussi les noyaux fongiques $(\mathrm{N})$ sont nombreux $(x 4000)$. 36. Hyphe d'Ascomycète (A) entre les cellules corticales jeunes (CC) de l'apex de la mycorhize ; les grains de Woronin $(W)$ permettent son identification (x 5000 ). 37. Hyphes intracellulaires d'Ascomycète (Ai) dans les jeunes cellules racinaires de l'apex de la mycorhize $(x 6000)$. Les traits correspondent à $1 \mu \mathrm{m}$. 
encore être présents mais sont apparemment peu actifs. De telles pénétrations sont fréquemment signalées dans d'autres ectomycorhizes (Atkinson, 1975 ; Giltrap, 1982 ; Harley et Smith, 1983 ; Godbout et Fortin, 1985 ; Kottke et Oberwinkler, 1986b ; Münzenberger et al, 1986 ; Wong et al, 1990). L'agression ne se fait jamais contre des cellules actives. II s'agirait donc d'un saprophytisme. Giltrap (1982) constate que la croissance de nombreuses espèces de lactaires n'est pas inhibée par des polyphénols (acides tannique et gallique). Ce comportement est comparable à celui des champignons saprophytes. Les lactaires en produisant des polyphénol-oxydases pénétreraient plus fréquemment que les autres champignons mycorhiziens dans les cellules corticales. Ceci est confirmé par Lindeberg (1948) qui a constaté de fortes réactions d'oxydation avec plusieurs espèces de lactaires dont $L$ flexuosus qui possède la même structure que $L$ blennius (Luppi et Gautero, 1967).

Dans l'ectomycorhize à $L$ subdulcis, un ascomycète est systématiquement observé dans tous les prélèvements des 2 stations. Brand $(1987,1993)$ signale également la

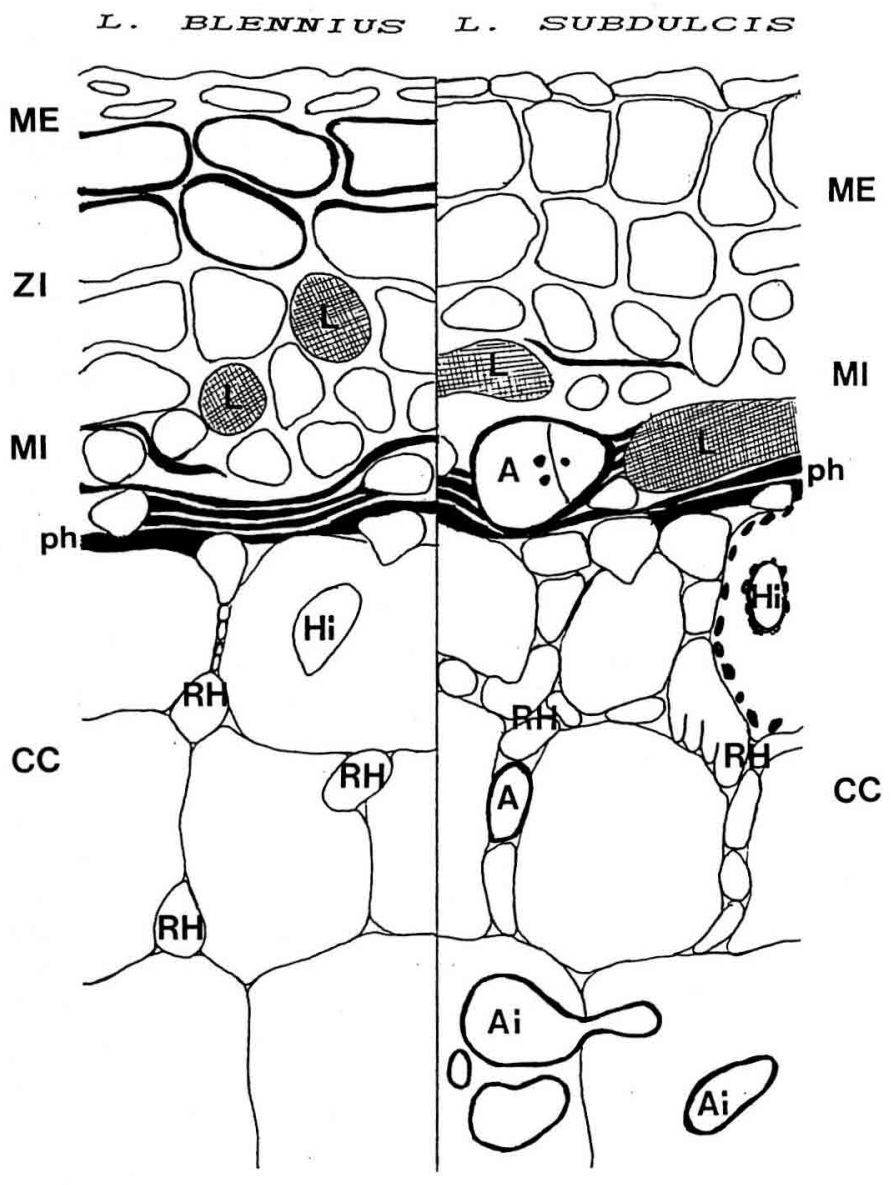

Fig 38. Ultrastructures comparées des mycorhizes à Lactarius blennius et $L$ subdulcis. 
présence d'un Ascomycète dans ce type de mycorhize. Il s'agit de Leucoscypha leucotricha, de la famille des Humariacées, qui colonise les litières forestières en décomposition (Dennis, 1968) et qui peut s'associer également avec les ectomycorhizes à $L$ rubrocinctus et à $L$ fuliginosus. Ces triples associations ne sont pas rares sur les racines de hêtre (Brand, 1987). Elles existent également chez les Conifères où l'on peut observer la présence simultanée de Chroogomphus ou Gomphidius avec Suillus ou Rhizopogon (Agerer, 1990). Cependant, il s'agit de 4 genres appartenant à la classe des Basidiomycètes, les 3 premiers relevant de l'ordre des Bolétales. Ce sont, dans ce cas, des champignons plus proches les uns des autres que les lactaires et les Ascomycètes.

L'Ascomycète ne peut apparemment pas être considéré comme un champignon symbiotique. Il peut coloniser des cellules vivantes à l'apex, mais il est beaucoup plus souvent observé dans des cellules mortes ou en voie de dégénérescence. II pourrait en revanche contribuer à favoriser l'établissement de l'ectomycorhize (Brand, 1993).

En conclusion, les 2 mycorhizes étudiées possèdent les caractéristiques des ectomycorhizes. Elles montrent cependant l'une et l'autre des structures spécifiques qui ne permettent pas de donner une structure type des mycorhizes de lactaires (fig 38).

L'ectomycorhize à $L$ blennius se caractérise par le développement d'un manteau qui apparaît particulièrement protecteur pour les cellules racinaires et par un réseau de Hartig plus lâche que celui de l'autre ectomycorhize.

La structure de l'ectomycorhize à $L$ subdulcis est plus classique. Cependant cette mycorhize se caractérise par la présence d'une autre souche fongique. Son abondant développement aux différents niveaux de la mycorhize reflète l'importance de ce champignon dont la présence doit modifier profondément la symbiose entre $L$ subdulcis et les racines du hêtre.

\section{RÉFÉRENCES}

Agerer R (ed) (1987) Colour Atlas of Ectomycorrhizae. Einhorm-Verlag, Schwäbisch Gmünd, Allemagne

Agerer R (1990) Studies on ectomycorrhizae XXIV. Ectomycorrhizae of Chroogomphus helveticus and $C$ rutilus (Gomphidiaceae, Basidiomycètes) and their relationship to those of Suillus and Rhizopogon. Nova Hedwigia 50, 1-63

Atkinson MA (1975) The fine structure of mycorrhizas. Thesis, Univ Oxford, Royaume-Uni

Blasius D, Feil W, Kottke I, Oberwinkler F (1986) Hartig net structure and formation in fully ensheathed ectomycorrhizas. Nord J Bot 6, 837-842

Brand $F$ (1987) A secondary fungal infection in mycorrhizae formed by Lactarius spp with Fagus sylvatica. In : Mycorrhizae in the next decade. Practical applications and research priorities (DM Sylvia, LL Hung, JH Graham, eds), Gainesville, FL, États-Unis, 189

Brand $F$ (1993) Mixed associations of fungi in ectomycorrhizal roots. In : Mycorrhizas in Systems. CAB Int (DJ Read et al, eds), Sheffield, Royaume-Uni, 142-147

Dennis RWG (1968) British Ascomycetes. J Cramer, Lehre, Royaume-Uni

Dop P, Gautier A (1928) Manuel de technique botanique, Histologie et Microbiologie. $2^{\mathrm{e}}$ ed, J Lamarre, Paris, France

de Faÿ E (1981) Histophysiologie comparée des écorces saines et pathologiques (maladie des encoches sèches) de l'Hevea brasiliensis. Thesis, univ Montpellier II, France

Giltrap NJ (1982) Production of polyphenol oxidases by ectomycorrhizal fungi with special reference to $\mathrm{Lac}$ tarius spp. Trans Br Mycol Soc 78, 75-81

Godbout C, Fortin JA (1985) Synthesised ectomycorrhizae of aspen: fungal genus level of structural characterization. Can J Bot 63, 252-262

Harley JL, Smith J (1983) Mycorrhizal symbiosis. Academic Press, London, Royaume-Uni

Kottke I, Oberwinkler F (1986) Mycorrhiza of forest trees: structure and function. Trees 1, 1-24

Kottke I, Oberwinkler F (1987) The cellular structure of the Hartig net: coenocytic and transfer cell-like organization. Nord $J$ Bot 7, 85-95

Lindeberg G (1948) On the occurrence of polyphenol oxidases in soil-inhabiting Basidiomycetes. Physiol Plant 1, 196-205 
Lison L (1960) Histochimie et cytochimie animale, principes et méthodes. vol II Gauthier-Villars, Paris, France

Luppi AM, Gautero C (1967) Ricerche sulle micorrize di Quercus rabur, $Q$ Petraea, $Q$ Pubescens in Piemonte. Allionia 13, 129-148

Marks GC, Foster RC (1973) Structure, morphogenesis and ultrastructure of ectomycorrhizae. In : Ectomycorrhizae. Their ecology and physiology (GC Marks, TT Kozlowski, eds), Academic Press, New York, États-Unis, 1-41

Massicotte HB, Peterson RL, Ackerley CA, Melville LH (1990) Structure and ontogeny of Betula alleghanniensis-Pisolithus tinctorius ectomycorrhizae. Can $J$ Bot 68, 579-593

Melville LH, Ackerley CA, Massicotte HB, Peterson RL (1988) An ultrastructural study of modifications in Dryas integrifolia and Hebeloma cylindrosporum during ectomycorrhiza formation. Bot Gaz 149, 408-418

Münzenberger $B$ (1991) Lösliche und zellwandgebundene Phenole in Mykorrihizen und nicht mykorrhizierten Wurzein der Fichte (Picea abies (L) Karst) und des Erdbeerbaumes (Arbutus unedo $L$ ) und ihre Bedeutung in der Pilz-Wurzeln-Interaktion. Thesis, univ Tübingen, Allemagne

Münzenberger B, Metzler B, Kottke I, Oberwinkler F (1986) Morphologische und anatomische Charak- terisierung der Mykorrhiza Lactarius deterrimusPicea abies in vitro. Z Mykol 52(2), 407-422

Prévost $A$ (1992) Contribution à l'étude cytophysiologique de la symbiose ectomycorhizienne chez les végétaux ligneux (Gymnospermes et Angiospermes). Thèse, univ Nancy I, France

Riedacker A (1981) 4. Physiologie de la croissance. 4.1. Croissance aérienne et souterraine. In : Le hêtre (E Teissier du Cros, ed), INRA, Paris, France, 160-169

Sterner O, Bergman R, Kihlberg J, Wickberg B (1985) The sesquiterpenes of Lactarius vellereus and their role in a proposed chemical defense system. J Nat Prod 48, 279-288

Voiry H (1981) Classification morphologique des ectomycorhizes du chêne et du hêtre dans le Nord-Est de la France. Eur J For Path 11 (5/6), 284-299

Wicklow DT (1988) Metabolites in the coevolution of fungal chemical defence systems. In: Coevolution of fungi with plants and animal (KA Pyrozynski, DL Hawksworth, eds), Academic Press, London, Royaume-Uni, 177

Wong KKY, Montpetit D, Piché Y, Lei J (1990) Root clonization by four closely related genotypes of the ectomycorrhizal basidiomycete Laccaria bicolor (Maire) Orton. Comparative studies using electorn microscopy. New Phytol 116, 669-679 